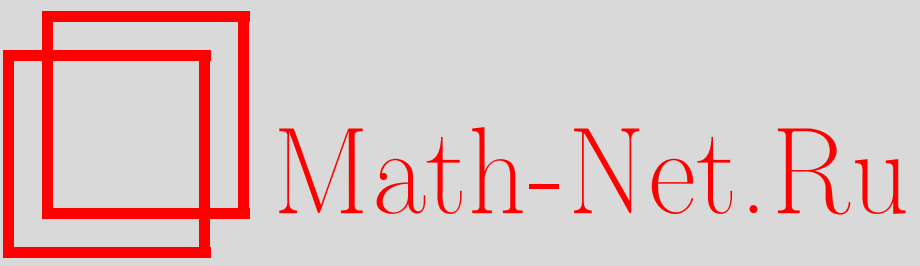

И. М. Гусейнов, Р. Т. Пашаев, Об одной обратной задаче для дифференциального уравнения второго порядка, УМН, 2002, том 57, выпуск 3, 147-148

DOI: https://doi.org/10.4213/rm517

Использование Общероссийского математического портала Math-Net.Ru подразумевает, что вы прочитали и согласны с пользовательским соглашением

http://www.mathnet.ru/rus/agreement

Параметры загрузки:

IP: 54.147 .182 .235

26 апреля 2023 г., 02:32:34 


\title{
ОБ ОДНОЙ ОБРАТНОЙ ЗАДАЧЕ ДЛЯ ДИФФЕРЕНЦИАЛЬНОГО УРАВНЕНИЯ ВТОРОГО ПОРЯДКА
}

\author{
И. М. ГУСЕЙНОВ, Р. Т. ПАШАЕВ
}

Рассмотрим граничную задачу

$$
\begin{gathered}
-y^{\prime \prime}+q(x) y=\lambda^{2} \rho(x) y, \quad 0<x<+\infty, \\
y(0)=0
\end{gathered}
$$

где функция $q(x)$ - вещественная,

$$
\int_{0}^{\infty} x|q(x)| d x<+\infty \text { и } \rho(x)= \begin{cases}\alpha^{2}, & 0 \leqslant x<a, \\ 1, & x \geqslant a .\end{cases}
$$

В случае $\alpha=1$, т.е. когда $\rho(x) \equiv 1$, обратная задача теории рассеяния для граничной задачи (1)-(2) полностью решена в работе [1] (см. также [2]-[4]). При $0<\alpha \neq 1$ эта задача изучалась в работе [5], однако в ней не было получено окончательных резултатов. В настоящей работе полностью решается обратная задача рассеяния для граничной задачи (1)-(2) в случае, когда $0<\alpha \neq 1$. Оказывается, что в данном случае разрыв у функции $\rho(x)$ сильно влияет на структуры представления решения Йоста и основного уравнения обратной задачи. Отметим, что подобные трудности не возникают для системы уравнений Дирака с разрывньми коэффициентами [6].

Как известно [5], граничная задача (1)-(2) имеет лишь конечное число простых отрицательных собственных значений $-\varkappa_{1}^{2}, \ldots,-\varkappa_{n}^{2}\left(\varkappa_{j}>0\right)$, а полуось составляет ее абсолютно-непрерывньй спектр. Для нормированных собственных функций задачи (1)-(2) при $x \rightarrow+\infty$ имеют место асимптотические формулы $u_{j}(x) \sim m_{j} e^{-\varkappa_{j} x}, j=1, \ldots, n ; u(x, \lambda) \sim e^{-i \lambda x}-$ $S(\lambda) e^{i \lambda x}$, в которых $S(\lambda),-\infty<\lambda<+\infty,-$ непрерьвная функция, обладающая свойством $\overline{S(\lambda)}=S(-\lambda)=S^{-1}(\lambda), m_{j}$ - некоторые положительные числа.

Совокупность величин $\left\{S(\lambda),-\infty<\lambda<+\infty ;-\varkappa_{1}^{2}, \ldots,-\varkappa_{n}^{2} ; m_{1}, \ldots, m_{n}\right\}$ называется данными рассеяния граничной задачи (1)-(2). Обратная задача теории рассеяния состоит в восстановлении граничной задачи $(1)-(2)$ (функции $q(x)$ ) по данным рассеяния.

Нетрудно проверить, что функция

$$
f_{0}(x, \lambda)=\frac{1}{2}\left(1+\frac{1}{\sqrt{\rho(x)}}\right) e^{i \lambda \mu^{+}(x)}+\frac{1}{2}\left(1-\frac{1}{\sqrt{\rho(x)}}\right) e^{i \lambda \mu^{-}(x)},
$$

где $\mu^{ \pm}(x)= \pm x \sqrt{\rho(x)}+a(1 \mp \sqrt{\rho(x)})$, является решением Йоста уравнения $(1)$, когда $q(x) \equiv 0$. В дальнейшем важную роль играет следующая теорема о представлении (нетреуголном) решения Йоста уравнения (1).

ТЕОРема 1. Если выполнены условия (3), то уравнение (1) имеет единственное решение $f(x, \lambda)$, представимое в виде

$$
f(x, \lambda)=f_{0}(x, \lambda)+\int_{\mu+(x)}^{+\infty} K(x, t) e^{i \lambda t} d t
$$

причем функция $K(x, \cdot) \in L_{1}\left(\mu^{+}(x),+\infty\right)$ и обладает следующими свойствами:
a) $\frac{d}{d x} K\left(x, \mu^{+}(x)\right)=-\frac{1}{4 \sqrt{\rho(x)}}\left(1+\frac{1}{\sqrt{\rho(x)}}\right) q(x)$,
b) $\frac{d}{d x}\left\{K\left(x, \mu^{-}(x)+0\right)-K\left(x, \mu^{-}(x)-0\right)\right\}=\frac{1}{4 \sqrt{\rho(x)}}\left(1-\frac{1}{\sqrt{\rho(x)}}\right) q(x)$,

если $q(x)$ дифференцируема, то $K(x, t)$ удовлетворяет (п.в.) уравнению
c) $\rho(x) K_{t t}^{\prime \prime}-K_{x x}^{\prime \prime}+q(x) K=0$
$0<x<\infty, \quad t>\mu^{+}(x)$. 
Метод восстановления $q(x)$ и решение обратной задачи основаны на том, что ядро $K(x, t)$ из представления (4) удовлетворяет следующему функционально-интегральному уравнению - основному уравнению обратной задачи:

$$
K(x, t)+\frac{1-\sqrt{\rho(x)}}{1+\sqrt{\rho(x)}} K(x, 2 a-t)+F(x, t)+\int_{\mu^{+}(x)}^{+\infty} K(x, \xi) F_{0}(\xi+t) d \xi=0, \quad t>\mu^{+}(x),
$$

где $F_{0}(x)$ и $F(x, t)$ определяются через данные рассеяния по формулам

$$
\begin{gathered}
F_{0}(x)=\frac{1}{2 \pi} \int_{-\infty}^{+\infty}\left\{S_{0}(\lambda)-S(\lambda)\right\} e^{i \lambda x} d \lambda+\sum_{j=1}^{n} m_{j}^{2} e^{-\varkappa_{j} x}, \quad S_{0}(\lambda)=\frac{\overline{f_{0}(0, \lambda)}}{f_{0}(0, \lambda)}, \\
F(x, t)=\frac{1}{2}\left(1+\frac{1}{\sqrt{\rho(x)}}\right) F_{0}\left(t+\mu^{+}(x)\right)+\frac{1}{2}\left(1-\frac{1}{\sqrt{\rho(x)}}\right) F_{0}\left(t+\mu^{-}(x)\right) .
\end{gathered}
$$

Теорема 2. Основное уравнение (5) имеет при каждом фиксированном $x \geqslant 0$ единственное решение $K(x, \cdot) \in L_{1}\left(\mu^{+}(x),+\infty\right)$.

СледСтвиЕ. Данные рассеяния однозначно определяют граничную задачу (1)-(2).

Использование уравнения (5) и теорем 1 и 2 позволяет установить характеристические свойства данных рассеяния:

Tеорема 3. Совокупность величин $\left\{S(\lambda),-\infty<\lambda<+\infty ;-\varkappa_{1}^{2}, \ldots,-\varkappa_{n}^{2} ; m_{1}, \ldots, m_{n}\right\}$, $0<\varkappa_{1}<\cdots<\varkappa_{n}, m_{j}>0, j=1,2, \ldots, n$, является данными рассеяния для некоторой граничной задачи вида (1)-(2) с коэффициентами из класса (3) в том и только том случае, когда

1) $S(\lambda)$ - непрерывная функция на вещественной оси $-\infty<\lambda<+\infty, \overline{S(\lambda)}=$ $S(-\lambda)=S^{-1}(\lambda)$

2) $S_{0}(\lambda)-S(\lambda)$ является преобразованием фурье некоторой функции из пространства $L_{1}(-\infty,+\infty)$, функиия $F_{0}(x)$ дифференцируема на $(2 a(1-\alpha),+\infty) u$ $\int_{0}^{\infty} x\left|F_{0}^{\prime}\left(2 \mu^{+}(x)\right)\right| d x<\infty$;

3) $\left.\frac{1}{2 \pi}\left\{\arg S_{0}(\lambda)-\arg S(\lambda)\right\}\right|_{+0} ^{+\infty}=n+\frac{1-S(0)}{4}$.

ЗАмечАниЕ. Аналогично исследуется обратная задача рассеяния для граничной задачи $(1)-(2)$ и в случае, когда $\rho(x)$ - положительная кусочно-постоянная функция с конечным числом точек разрыва.

Авторы благодарны проф. М. Г. Гасымову за полезное обсуждение.

\section{СПИСОК ЛИТЕРАТУРЫ}

[1] В. А. Марченко // Докл. АН СССР. 1955. Т. 104. № 5. С. 695-698. [2] В. А. Марченко. Операторы Штурма-Лиувилля и их приложения. Киев: Наукова думка, 1977. [3] М. Г. Крейн // Докл. АН СССР. 1955. Т. 105. С. 433-436. [4] Б. М. Левитан // Матем. заметки. 1975. Т. 17. № 4. С. 611-624. [5] М. Г. Гасымов // Неклассические методы в геофизике. Новосибирск: Наука, 1977. С. 37-44. [6] И. М. Гусейнов // Докл. АН Азерб. 1999. Т. 55. № 1-2. С. 13-18. 